\title{
Colloid Electrophoresis for Strong and Weak Ion Diffusivity
}

\author{
Giovanni Giupponi* and Ignacio Pagonabarraga $^{\dagger}$ \\ Departament de Fisica Fonamental, Universitat de Barcelona, Carrer Martí i Franques, 08028 Barcelona, Spain
}

(Received 10 December 2010; published 17 June 2011)

\begin{abstract}
We study the electrophoretic flow of suspensions of charged colloids with a mesoscopic method that allows us to model generic experimental conditions. We show that for highly charged colloids their electrophoretic mobility increases significantly and displays a mobility maximum on increasing the colloidal charge for all salt concentrations. The electrophoretic mobility of highly charged colloids is also enhanced significantly when ion advection is dominant, leading to a strong heterogeneity in the local electrophoretic response especially at low-salt concentration, when ion diffuse layers overlap.
\end{abstract}

PACS numbers: 47.57.jd, 47.11.St, 47.65.- d

Effective electrostatic interactions between charged colloidal particles in solution play a fundamental role in determining the macroscopic phase and rheological properties of colloidal suspensions that are pivotal for many applications in material science $[1,2]$. In particular, the response of these systems to applied electric fields enhances the degree of experimental control and tunability of suspensions down to the nanoscale and provides a natural means to design and operate nanodevices. As a result, electrokinetic techniques are pragmatically exploited to control fluid flow at small scales, as, for example, in nanofluidic molecular sorting [3,4], nanomedicine protocols [5], or micropattern assembly [6].

A theoretical and quantitative knowledge of electrophoresis is still not complete, as understanding the electrophoretic flow (EF) of the solvent and the charged components (ionized macromolecules, counterions, and salt ions) requires the combined treatment of solvent flow coupled to the diffusion and advection of the dissolved electrolyte in the presence of charged colloids. The dynamical coupling between suspended charged components through hydrodynamics, leading to effective electrostatic interactions, does not allow for exact solutions; analytic predictions are restricted to the linear regime, and either numerical or simulation studies are required to assess the interplay between electrostatics and hydrodynamics that results in the EF. The relevant length (time) scales involved in EF range from the nanometer (nanosecond), characteristic of the charge distribution around the particles, to the colloidal size itself, up to the micrometer (microsecond). This disparity of scales complicates the computational modeling because molecular dynamics is practically limited to the detailed resolution of the smallest nanoscales. Mesoscopic modeling [7-9], which includes the appropriate solvent dynamics at a coarse scale, overcomes the difficulties of handling multiscale simulations of a charged colloidal suspension EF. Mesoscopic modeling has been recently used to study the nonequilibrium dynamics of a variety of complex charged heterogeneous systems [10] and, therefore, offers a valuable way to obtain theoretical predictions for a comprehensive $\mathrm{EF}$, in primis the fundamental EF of a charged spherical particle.

In this Letter, we study the electrokinetic response of charged colloids and examine the relevance of nonlinear coupling and ion specificity for different types of EF due to charge density deformations induced by applied electric fields. We do so by taking advantage of a novel mesoscopic simulation technique that naturally includes appropriate boundary conditions and does not require any assumption beyond Poisson-Boltzmann. We then capture the significant physical couplings between charges, colloids, and solvent, accounting for the nonlinear response of the electrolyte rearrangements to the applied field. Moreover, our model can be used under general experimental conditions of salt concentration, ion diffusivity, and colloid surface charge density.

A charged macromolecule in solution is surrounded by a counterion and salt ion cloud, which screens the colloidal charge depending on the salt concentration $c_{s}$ and the solvent dielectric properties. Applying an electric field $E$ causes the electrolyte and macromolecule to move. The limiting particle velocity at the steady state, $v_{l}$, develops as a result of the balance between the electrostatic and viscous forces. However, the deformed charge distribution of coions and counterions around the macromolecule, which gives rise to an electric double layer (EDL) around the object, is hard to derive analytically even in thermodynamic equilibrium, and usually solutions are known for low electrostatic potentials at the colloid surface (referred to as the $\zeta$ potential), when linear electrostatics holds $[1,11]$. Moreover, nonlinear EDL distortion due to the external electric field leads to further difficulties in its analytic understanding.

O'Brien and White (OW) [12] have integrated numerically the electrokinetic equations for the $\mathrm{EF}$ of an infinitely diluted spherical colloid in the linear regime. Their results indicate that the particle electrophoretic mobility $\mu=$ $v_{l} / E$ depends on the colloid radius $a$, salt concentration $c_{s}$, and dielectric properties of the solvent at temperature $T$ through the Debye screening length $\lambda_{D}=\left(8 \pi l_{B} c_{s} z_{+}^{2}\right)^{-1 / 2}$ 
[for a symmetric electrolyte of valence $z_{+}$, here $z_{+}= \pm 1$, in a solvent with Bjerrun length $l_{B}=e^{2} /\left(4 \pi \epsilon k_{B} T\right)$ [11], with $e$ the electron charge and $k_{B}$ the Boltzmann factor] and $\zeta$, predicting either mobility saturation or a maximum for high- $\zeta$ values. Using different mesoscopic models, Kim, Nakayama, and Yamamoto [13] were able to confirm OW predictions [12] for low $c_{s}$, while Lobaskin et al. $[14,15]$ showed that the electrophoretic behavior of salt-free systems can be systematically mapped to a corresponding low-salt suspension. Both cases, however, address the restricted case of small $\zeta$ and $c_{s}$, where OW predictions are expected to hold.

Our mesoscopic representation of charged colloidal suspensions builds on a continuous description of the electrolyte, characterized in terms of the anion and cation local densities $\rho_{ \pm}$. These densities evolve according to the electrokinetic equations, which read

$$
\begin{gathered}
\frac{\partial \rho_{k}}{\partial t}=-\vec{\nabla} \cdot \rho_{k} \vec{v}+\vec{\nabla} \cdot D_{k}\left[\vec{\nabla} \rho_{k}+e \beta z_{k} \rho_{k} \vec{\nabla} \varphi\right], \\
\frac{\partial \rho \vec{v}}{\partial t}=\eta \nabla^{2} \vec{v}-\vec{\nabla} p_{i d}+\beta \sum_{k} e z_{k} \rho_{k} \vec{\nabla} \varphi, \\
\nabla^{2} \varphi=-\frac{1}{\epsilon} \sum_{k} e z_{k} \rho_{k},
\end{gathered}
$$

where $D_{k}, z_{k}, k=+,-$ are the diffusivities and valences of positive and negative ions, $\rho, \vec{v}, p_{i d}$, and $\eta$ correspond to the solvent density, velocity, ideal pressure, and shear viscosity, respectively, $\varphi$ is the electrostatic potential, and $\epsilon$ is the solvent permittivity. Equation (1) expresses ion mass conservation as a result of diffusion and advection, while the solvent motion [Eq. (2)] evolves according to the Navier-Stokes equation for a viscous fluid accelerated by electrostatic forces due to local charge density. Finally, the Poisson equation enforces the electrostatic coupling between the charged species and the macromolecules.

The solvent motion emerges from a discrete lattice formulation of Boltzmann's kinetic equation [8] coupled to a discrete solution of the convection-diffusion equation for the dissolved charged ion species [16]. Hence, we regard the counterions and salt ions as scalar fields within the Poisson-Boltzmann level [11]. The colloidal macromolecules are individually resolved, embedded on the lattice, and coupled to the fluid through appropriate kinetic rules applied on their boundaries [17]. The hydrodynamic forces exerted by the fluid on the suspended particles, together with the electrostatic and dispersion forces, determine the motion of the macromolecules in the fluid [18]. The finite resolution of the colloidal particles on a lattice requires a proper calibration to identify the effective size where $\zeta$ is consistent with the colloidal charge [19]. The electrostatic potential drop around the colloids emerges consistently as a result of the ionic dynamics coupled to the fluid flow without further assumptions. Although such an approach disregards ion correlations, it provides a general framework to address electrokinetics at weak and strong couplings and identifies the relevant competing physical mechanisms in charged driven fluids.

We start by analyzing the electrophoretic mobility $\mu$ of a crystal of spherical colloids of charge $Z e$ and radius $a$ as a function of $c_{s}, \zeta$, and volume fraction $\Phi=4 \pi a^{3} / 3 L^{3}$, where $L$ stands for the system size. In order to compare with experimental and numerical results, we introduce the dimensionless mobility $\tilde{\mu}=\frac{6 \pi \mu \eta l_{B}}{e}$ and $\zeta$ potential $\tilde{\zeta}=\frac{e \zeta}{k_{B} T}$. The units in the simulations ensure that the relevant scales keep the right ordering and are properly resolved on the lattice [20]. We take $\kappa a=0.5$ and 8.0, with $\kappa=1 / \lambda_{D}$, representative of low- to high-salt concentrations [12], covering 2 orders of magnitude in molarity, $\sim 10^{-4}-10^{-2} M$, when mapping our simulation systems to polystyrene spheres with a radius of $17 \mathrm{~nm}$ [21]. The applied electric field is tuned to remain in the linear response regime $E \ll \tilde{\zeta} / \lambda_{D}$.

Figure 1 shows the dimensionless mobility $\tilde{\mu}$ against $\tilde{\zeta}$. For small values $\tilde{\zeta} \lesssim 3$, we observe an excellent agreement with the OW theoretical prediction [12], which holds in the linear regime for low values of $\tilde{\zeta}$. Our data show that increasing $\tilde{\zeta}$ leads to an enhancement of $\tilde{\mu}$ with respect to OW. Such a deviation increases with $\tilde{\zeta}$ and with the amount of charge in the system up to $50 \%$ for narrow double layers, $\kappa a=8.0$. Although the theoretical predictions from OW display a maximum outside the linear regime and only for thin double layers $(\kappa a \gtrsim 2.75)$, the maxima observed in Fig. 1 appear at higher- $\zeta$ potentials and develop for any $\kappa a$. Therefore, these results indicate that care has to be taken when using OW curves to compare with experimental results.

The model described reproduces quantitatively experimental data for low- $\tilde{\zeta}$, salt-free colloidal suspensions. In Fig. 2, we show that agreement with experimental data is obtained for latex particles suspended in water [14,21] for colloid volume fractions $\Phi \gtrsim 0.001$. For very low $\Phi$, the

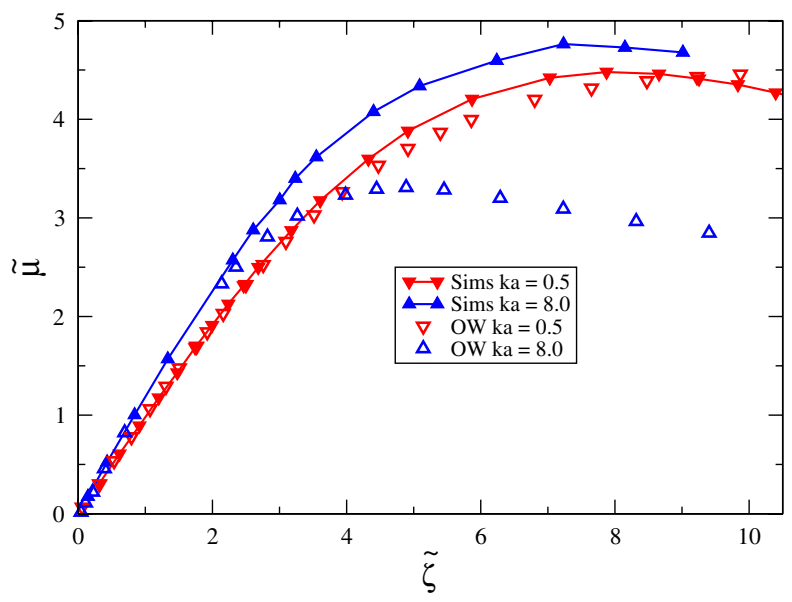

FIG. 1 (color online). Dimensionless electrophoretic mobility for a spherical colloidal particle, $\tilde{\mu}$, versus the dimensionless $\zeta$ potential $\tilde{\zeta}$. Results from simulations (filled symbols) and OW [12] (empty symbols) for $k a=0.5$ (0.8), triangles down (up). 


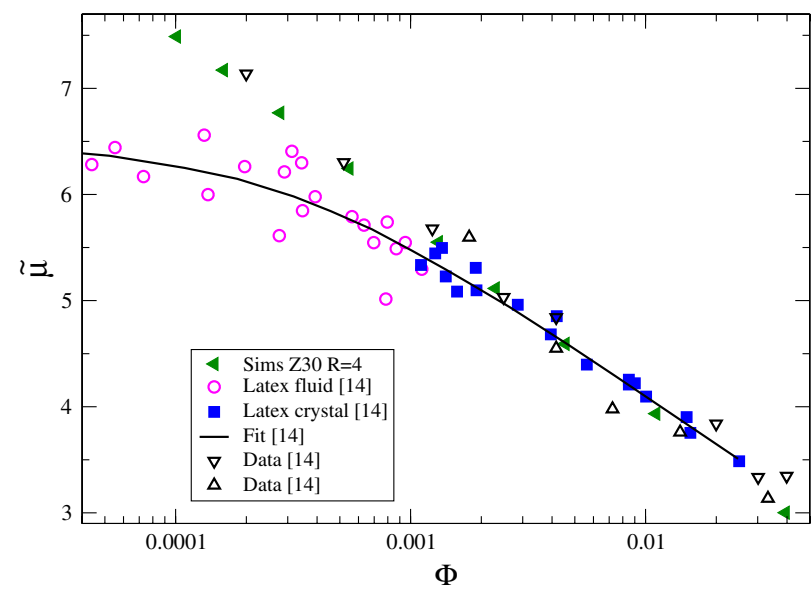

FIG. 2 (color online). Dimensionless electrophoretic mobility $\tilde{\mu}$ for a colloidal particle with charge $Z=30$ and radius $a=4.0$ versus colloid volume fraction $\Phi$ (triangle left). No salt is added to the system. Experimental (sphere and square symbols) and simulation results (empty triangles) from Lobaskin et al. [14].

ionic contribution due to water dissociation is relevant [14] and the mobility dependence on $\Phi$ vanishes. Figure 2 also displays simulation results where counterions are resolved individually [14]. The agreement shows that the electrophoretic response observed experimentally can be captured through the counterion density and that ionic finite-size electrostatic correlations are subdominant.

Even if in many situations individual ion resolution is irrelevant, its diffusion may affect significantly the electrokinetic response of charged colloids. We have analyzed the effect of ion diffusion for symmetric monovalent electrolytes, $D=D_{ \pm}$, and have quantified the importance of electrolyte diffusion to flow advection in terms of the Péclet number $\mathrm{Pe}=a v_{0} / D$, where the characteristic fluid velocity is induced by the colloid and reads $v_{0}=$ $e Z E / 6 \pi \eta a$. Figure 3 shows the dimensionless steady state velocity $v_{l} / v_{0}$ for a colloidal crystal at $\Phi \sim 10^{-2}$ as a function of Pe for different salt concentrations $c_{s}, \kappa a=$ $0.5,1.0,8.0$, covering from narrow to wide EDL at a fixed colloidal charge density $\tilde{\zeta} \sim 5.0$ in the nonlinear regime. The results show that for strong ion diffusivity the mobility is not affected by advection, while at larger Pe a significant increase in the colloidal mobility, departing from the high diffusivity regime at $\mathrm{Pe}=0$, is observed. The inset in Fig. 3, where we display $\tilde{\mu}$ as a function of the colloidal $\tilde{\zeta}$ for regimes where ion diffusivity is either subdominant or relevant, indicates that the competition between ion diffusion and advection by the incoming flow becomes quantitatively significant in the nonlinear regime. The mobility enhancement reported at small electric fields arises from ion advection, therefore differing from the mobility enhancement associates to charge stripping under strong applied fields [22]. Although we cannot discard that a maximum in $\tilde{\mu}$ develops asymptotically, for the range of $\tilde{\zeta}$ accessible experimentally, $\tilde{\zeta} \lesssim 10[1,12]$, we do not observe a saturation of $\tilde{\mu}$. Results in Figs. 1 and 2

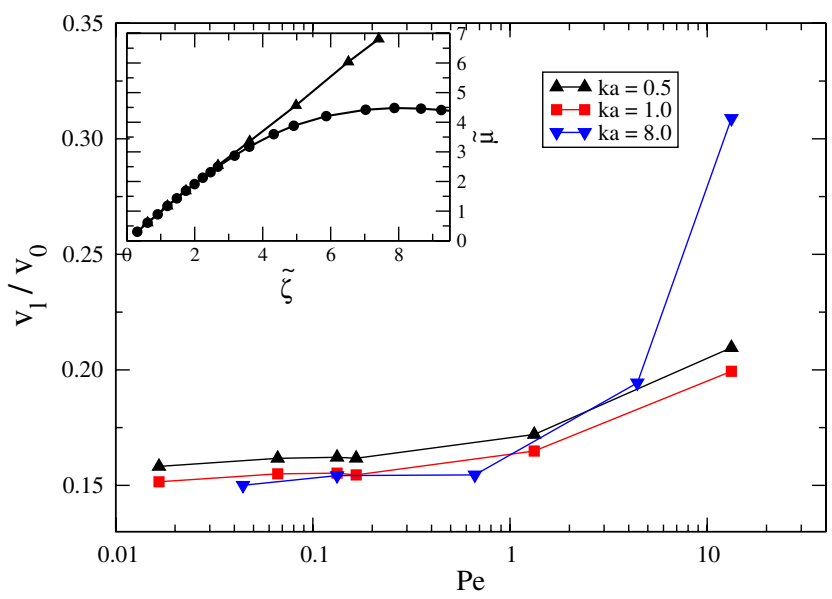

FIG. 3 (color online). Steady state colloidal velocity $v_{l} / v_{0}$ versus Péclet flow number for $\kappa a=0.5,1.0,8.0$. Inset: Dimensionless mobility $\tilde{\mu}$ versus dimensionless $\zeta$ potential $\tilde{\zeta}$, for $\kappa a=0.5, \mathrm{Pe} \sim 10^{-3}$ (filled circles), and $\mathrm{Pe} \sim 0.3$ (filled triangles).

correspond to the small $\mathrm{Pe} \sim 10^{-3}$ regime to compare with theories and experiments where diffusivity is dominant; however, the results of Fig. 3 indicate that diffusion can become a relevant variable because a wide range of Pe can be reached experimentally. For example, for a colloidal particle of radius $a \sim 0.1 \mu \mathrm{m}$, charge density $\sigma \sim 8 \cdot 10^{-2} \mathrm{C} / \mathrm{m}^{2}$, and an external field $E_{\text {ext }} \sim$ $10^{3} \mathrm{~V} / \mathrm{m}$ [21], $\mathrm{Pe} \sim 10^{-1}$ [1] for an electrolyte composed of small ions with typical diffusivity $D \sim 10^{-9} \mathrm{~m}^{2} / \mathrm{s}$, Pe can nevertheless increase 1 or 2 orders of magnitude imposing strong electric fields or in the presence of bigger, nanosized ions [23], which induce a greater EDL disruption. To characterize the EDL degree of distortion as a function of $\mathrm{Pe}$, we compute the dimensionless eigenvalues $\tilde{\lambda}$ of the charge density inertia tensor around the colloid center, $\tilde{\lambda}_{i}=\lambda_{i} /\left(Z a^{2}\right), i=x, y, z$. If we apply the external electric field along the $x$ axis, the axisymmetric structure of the EDL gives an asymmetry of the transverse eigenvalue as Pe increases. Table I reports the magnitude of the three eigenvalues for different values of Pe and $c_{s}$. For all salt concentrations $\tilde{\lambda}_{i}$ increase with Pe, showing a larger spread of the EDL that results in a lower screening of the colloid charge, therefore raising its mobility at steady state. The different values of $\tilde{\lambda}_{i}$ show that the axisymmetric charge distribution around the colloid develops a significant departure from isotropy only when ion advection drag becomes dominant, at experimentally high Pe.

TABLE I. Charge inertia matrix eigenvalues.

\begin{tabular}{cccccc}
\hline \hline$\kappa a$ & $\mathrm{Pe}$ & $\tilde{\lambda}_{x}$ & $\tilde{\lambda}_{y}$ & $\tilde{\lambda}_{z}$ & $\tilde{\mu}$ \\
\hline 0.5 & $2.54 \cdot 10^{-3}$ & 1.626848 & 1.627185 & 1.627185 & 4.42 \\
0.5 & $3.12 \cdot 10^{-1}$ & 1.676013 & 1.676420 & 1.676420 & 6.81 \\
8.0 & $6.63 \cdot 10^{-3}$ & 0.756485 & 0.756834 & 0.756834 & 4.34 \\
8.0 & $8.60 \cdot 10^{-1}$ & 0.764143 & 0.764346 & 0.764346 & 5.62 \\
\hline \hline
\end{tabular}




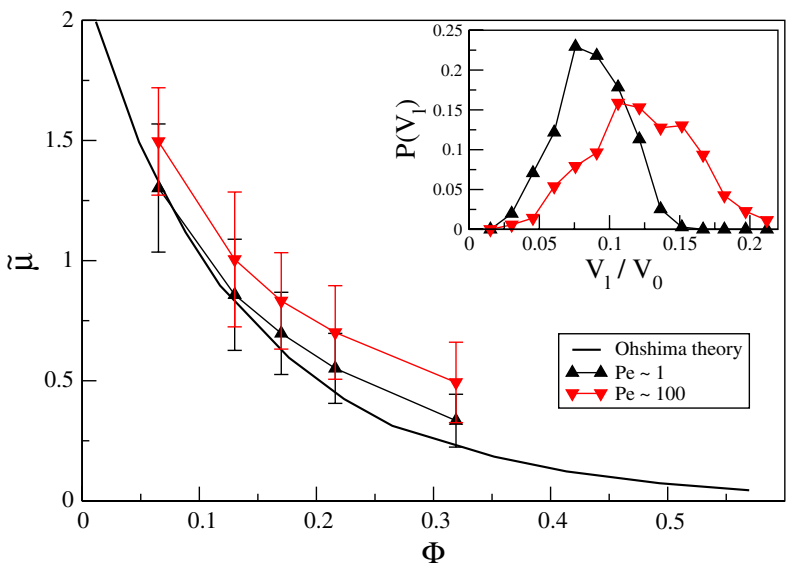

FIG. 4 (color online). Dimensionless mobility $\tilde{\mu}$ versus volume fraction $\Phi$ for a suspension of particles with overlapping EDLs, $\mathrm{Pe} \sim 1,10^{2}$. Inset: Probability distribution of particle velocities $v_{l}$ when $\Phi=0.32, \kappa a=1.0$ (overlapping EDLs).

Finally, we study colloidal suspensions at low-salt concentrations and at volume fractions when EDLs overlap. We compare our results for $\tilde{\zeta}=2.0$ with analytical predictions derived by Ohshima using single-particle charge distributions and linear electrostatics [24]. Figure 4 shows that deviations from Ohshima's theory develop for $\Phi \simeq 0.1$, in agreement with the theoretical expectation that EDL overlap for $\Phi \geq \kappa a /(1+\kappa a)^{3}$. We unveil also the fundamental role played by advection-diffusion competition in EF, as Pe leads to an increase in the electrophoretic mobility. Moreover, ion diffusivity is seen to alter conjointly the dispersion of $v_{l}$ in the linear regime for significant EDL overlap, as depicted in the inset in Fig. 4. Understanding the physical mechanisms underlying the increase in $\tilde{\mu}$ with $\Phi$ for highly diluted suspensions with overlapping EDLs [21], $\Phi<10^{-3}$, requires further investigation beyond current computational capabilities. The probability distribution function of colloid velocities for $\Phi=0.32, \kappa a=1.0$ shows that on increasing Pe not only does the mean $v_{l}$ shift to larger values but also the probability distribution function widens. As a result, an increase in ion drag due to EF leads to larger spread in local $v_{l}$, enhancing the colloidal local electrophoretic response heterogeneity.

In summary, we have comprehensively studied the electrokinetic response of suspensions of charged colloids, opening up new possibilities to fine-tune and extend current electrokinetic techniques. We have shown that the colloidal mobility develops a maximum due to the competition between EDL distortion and the EF induced by the applied electric field. We have revealed the importance of ion diffusivity in identifying an experimental regime of enhanced mobility and increased heterogeneity of local electrophoretic response when the external driving dominates over ion diffusion. At small ion diffusivities, the overlap of EDL leads to a strong dynamic coupling between colloids, causing strong heterogeneities in the colloidal response.

We acknowledge J. Faraudo for fruitful discussions, the IEF Marie Curie scheme (G. G.), and the Dirección General de Investigación (Spain) and DURSI project (I. P.) for financial support under Projects No. FIS 200804386 and No. 2009SGR-634, respectively.

*giupponi@ffn.ub.es

ipagonabarraga@ub.edu

[1] W. B. Russel, D. A. Saville, and W. R. Schowalter, Colloidal Dispersions (Cambridge University Press, Cambridge, England, 1991).

[2] R. G. Larson, The Structure and Rheology of Complex Fluids (Oxford University, New York, 1999).

[3] W. Sparreboom, A. Van Den Berg, and J.C.T. Eijkel, Nature Nanotech. 4, 713 (2009).

[4] R. Austin, Nature Nanotech. 2, 79 (2007).

[5] F. Andre and L. M. Mir, Gene Therapy 11, S33 (2004).

[6] R. C. Hayward, D. A. Saville, and I. A. Aksay, Nature (London) 404, 56 (2000).

[7] P. J. Hoogerbrugge and J. Koelman, Europhys. Lett. 19, 155 (1992).

[8] R. Benzi, S. Succi, and M. Vergassola, Phys. Rep. 222, 145 (1992).

[9] A. Malevanets and R. Kapral, J. Chem. Phys. 110, 8605 (1999).

[10] I. Pagonabarraga, B. Rotenberg, and D. Frenkel, Phys. Chem. Chem. Phys. 12, 9566 (2010).

[11] J. Barrat and J. Hansen, Basic Concepts for Simple and Complex Liquids (Cambridge University Press, Cambridge, England, 2003).

[12] R. W. O'Brien and L. R. White, J. Chem. Soc., Faraday Trans. 2 74, 1607 (1978).

[13] K. Kim, Y. Nakayama, and R. Yamamoto, Phys. Rev. Lett. 96, 208302 (2006).

[14] V. Lobaskin et al., Phys. Rev. Lett. 98, 176105 (2007).

[15] B. Dünweg et al., J. Phys. Condens. Matter 20, 404214 (2008).

[16] F. Capuani, I. Pagonabarraga, and D. Frenkel, J. Chem. Phys. 121, 973 (2004).

[17] A. J. C. Ladd, J. Fluid Mech. 271, 285 (2006).

[18] B. Rotenberg, I. Pagonabarraga, and D. Frenkel, Faraday Discuss. 144, 223 (2010).

[19] G. Giupponi and I. Pagonabarraga, Phil. Trans. R. Soc. A 369, 2546 (2011).

[20] M. Cates et al., J. Phys. Condens. Matter 16, S3903 (2004).

[21] N. Garbow et al., J. Phys. Condens. Matter 16, 3835 (2004); M. Medebach and T. Palberg, ibid. 16, 5653 (2004); T. Palberg et al., ibid. 16, S4039 (2004).

[22] M. Fixman and S. Jagannathan, Macromolecules 16, 685 (1983).

[23] A. Martín-Molina et al., Soft Matter 5, 1350 (2009).

[24] H. Ohshima, Theory of Colloid and Interfacial Electric Phenomena (Academic, New York, 2006). 